\title{
Chronic inflammation upregulates chemokine receptors and induces neutrophil migration to monocyte chemoattractant protein-1
}

\author{
Brent Johnston, ${ }^{1}$ Alan R. Burns, ${ }^{2}$ Makoto Suematsu, ${ }^{3}$ Thomas B. Issekutz, ${ }^{4}$ \\ Richard C. Woodman, ${ }^{1}$ and Paul Kubes ${ }^{1}$ \\ ${ }^{1}$ Immunology Research Group, Department of Physiology and Biophysics, University of Calgary, Calgary, \\ Alberta T2N 4N1, Canada \\ ${ }^{2}$ Department of Medicine, Section of Cardiovascular Sciences, Baylor College of Medicine, Houston, Texas 77030, USA \\ ${ }^{3}$ Department of Biochemistry, Keio University School of Medicine, Tokyo 160-8582, Japan \\ ${ }^{4}$ Department of Pediatrics, Division of Immunology, Rheumatology and Infectious Diseases, Dalhousie University, \\ Halifax, Nova Scotia B3J 3G9, Canada \\ Address correspondence to: Paul Kubes, Immunology Research Group, Department of Physiology and Biophysics, \\ Faculty of Medicine, The University of Calgary, Calgary, Alberta T2N 4N1, Canada. Phone: (403) 220-8558; \\ Fax: (403) 283-1267; E-mail: pkubes@acs.ucalgary.ca
}

Received for publication September 14, 1998, and accepted in revised form March 26, 1999.

\begin{abstract}
Monocyte chemoattractant protein-1 (MCP-1) is a CC chemokine that stimulates monocyte recruitment when injected into tissues of healthy animals. However, the function of this chemokine in models with preexisting inflammation is not known. Therefore, MCP-1 was superfused over the mesentery of naive rats or rats with chronic adjuvant-induced vasculitis. MCP-1 elicited increased leukocyte transendothelial migration in adjuvant-immunized rats compared with naive animals. Surprisingly, histology revealed that neutrophils constituted the majority of leukocytes recruited in adjuvant-immunized animals. In vitro, MCP-1 was also able to induce chemotaxis of neutrophils isolated from adjuvant-immunized rats but not from naive rats. Flow cytometry revealed novel expression of the $\mathrm{CC}$ chemokine receptors CCR1 and CCR2 on neutrophils from adjuvant-immunized animals. In naive animals, an antibody against CD18 blocked leukocyte adhesion and emigration in response to MCP-1. In adjuvant-immunized animals, leukocyte adhesion was reduced by antibodies against the $\alpha_{4}$-integrin but not by antibodies against CD18. However, the CD18 antibody did block emigration. To our knowledge, this study is the first to show increased sensitivity to a CC chemokine in a model with preexisting inflammation, and altered leukocyte recruitment profiles in response to MCP-1. It also demonstrates that CD18 is required for chemokine-induced leukocyte transendothelial migration, independent of its known role in mediating firm adhesion.
\end{abstract}

J. Clin. Invest.103:1269-1276 (1999).

\section{Introduction}

Leukocyte recruitment from the vasculature to sites of inflammation is dependent on a multistep cascade of adhesive interactions $(1,2)$. Leukocytes within the main stream of blood initially tether to the endothelium and engage in transient adhesive interactions that manifest as leukocyte rolling. Under acute inflammatory conditions, the tethering and rolling steps are mediated primarily by the selectin family of adhesion molecules (L-selectin, Eselectin, and P-selectin) (2-5). However, there is a growing body of evidence showing that the $\alpha_{4}$-integrins $\left(\alpha_{4} \beta_{1}\right.$ and $\alpha_{4} \beta_{7}$ ) can mediate tethering and rolling of isolated eosinophils and mononuclear leukocytes (6-8), as well as leukocyte rolling under subacute and chronic inflammatory conditions in vivo $(3,5,9)$. Rolling leukocytes may become activated and adhere firmly to the endothelium via the $\alpha_{4}$-integrins and/or the $\beta_{2}$-integrins (CD11 and CD18) $(2,9,10)$. If an appropriate chemotactic gradient is present, firmly adherent leukocytes may then transmigrate across the vessel wall to sites of potential injury. There are several types of chemotactic factors that can elicit leukocyte adhesion and chemotaxis. These include lipid mediators $(11,12)$, complement components $(13)$, bacterial products $(3,14)$, and chemokines $(10,15,16)$.

Monocyte chemoattractant protein-1 (MCP-1), a member of the CC family of chemokines, has been studied extensively. In vitro, MCP-1 is chemotactic for monocytes and lymphocytes but not neutrophils (17-19). In vivo, MCP-1 elicits monocyte infiltration when injected into rat skin or the skin or peritoneum of mice (20-22). Similarly, transgenic mice exhibit increased monocyte and lymphocyte accumulation at sites of MCP-1 overexpression $(23,24)$. Monocytes and lymphocytes express the chemokine receptor CCR2, which is a high-affinity receptor for MCP-1 (25-27). In CCR2-deficient mice, monocyte recruitment to sites of inflammation is impaired, while neutrophil recruitment remains intact $(16,28)$. However, it is not known whether this chemotactic profile is maintained in a situation where a chronic inflammatory disease is already present.

We have previously characterized the development of a vasculitis in the mesenteric venules of rats immunized with Mycobacterium butyricum in Freund's mineral oil adjuvant $(3,9)$. In this model, there is a marked increase 
$\mathbf{a}$
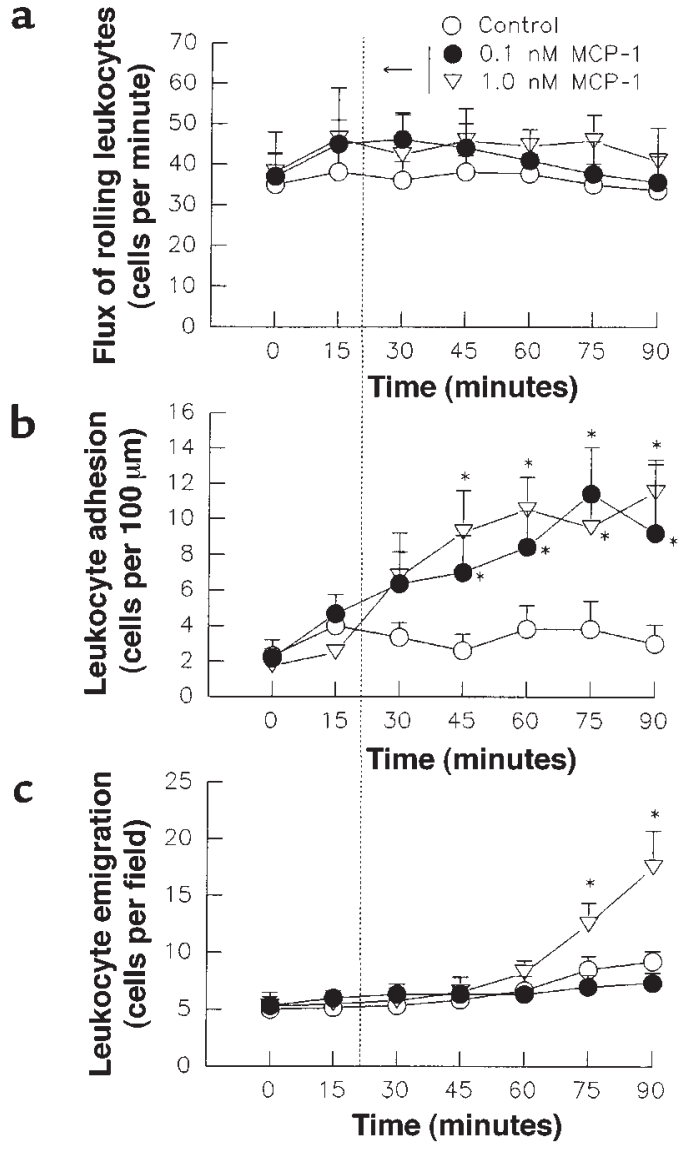

Figure 1

Leukocyte rolling flux (a), adhesion (b), and emigration (c) in mesenteric postcapillary venules of naive rats superfused with control buffer or the CC chemokine MCP-1. Following an initial 20-minute control period, during which the mesentery was superfused with bicarbonate-buffered saline, the mesentery was superfused for 70 minutes with buffered saline (control, $n=6)$ or buffered saline containing $0.1(n=6)$ or $1.0(n=4) \mathrm{nM}$ $\mathrm{MCP}-1 .{ }^{*} P<0.05$ vs. untreated control group.

in the number of rolling and firmly adherent leukocytes within the vasculature. In contrast to acute models of inflammation where leukocyte recruitment is dependent primarily on P-selectin and the $\beta_{2}$-integrins $(2-4,10)$, Lselectin and the $\alpha_{4}$-integrin are the major contributors to the rolling and adhesion observed in adjuvantinduced vasculitis $(3,9)$. Despite the increased leukocyte trafficking in mesenteric venules, there is very little leukocyte infiltration into the extravascular connective tissue of these animals. This allows us the opportunity to examine the action and mechanisms of chemokines in a preexisting inflammatory state.

The purpose of this study was to examine the kinetics and mechanisms of MCP-1-induced leukocyte recruitment under normal baseline and preexisting inflammatory conditions. MCP-1 was superfused over the mesentery of naive rats or rats with chronic adjuvant-induced vasculitis. We show that sensitivity to MCP-1 was increased at least 100-fold in the chronic inflammatory setting. Additionally, we show a shift in the leukocyte recruitment profile in response to MCP-1. Selectivity of MCP-1 for mononuclear cell populations was lost in adjuvant-immunized rats, and the majority of recruited leukocytes were neutrophils. This was associated with increased expression of the chemokine receptors CCR1 and CCR2 on neutrophils from adjuvant-immunized rats, and increased neutrophil chemotaxis in response to MCP-1 in vitro. Finally, adhesion in the adjuvant-induced model was dependent on the $\alpha_{4}$-integrin, while emigration could be inhibited with a CD18 antibody. To our knowledge, this demonstrates for the first time that CD18 is required for chemokine-induced leukocyte transendothelial migration, independent of its known role in mediating firm adhesion.

\section{Methods}

Adjuvant immunization. Under light anesthetic (diethyl ether; BDH Inc., Toronto, Ontario, Canada), male Sprague-Dawley rats (150-200 g) were injected subcutaneously at the base of the tail with a solution of $0.75 \mathrm{mg}$ of heat-killed M. butyricum (Difco Laboratories, Detroit, Michigan, USA) in $0.1 \mathrm{~mL}$ Freund's mineral oil adjuvant (Difco Laboratories). A 10-fold increase in leukocyte trafficking through mesenteric postcapillary venules has been characterized previously between 4 and 20 days after $M$. butyricum immunization $(3,9)$. Animals were used 12-14 days after immunization.

Intravital microscopy. Rats were maintained on a purified laboratory diet and fasted for 18-24 hours prior to surgery. Animals were anesthetized with an intraperitoneal injection of pentobarbital sodium $(55 \mathrm{mg} / \mathrm{kg}$ body weight). The right carotid artery was cannulated to monitor systemic arterial blood pressure (model P23XL pressure transducer, Viggo-Spectramed, Oxnard, California, USA; and model 7 physiologic recorder, Grass Instrument Co., Quincy, Massachusetts, USA). The right jugular vein was cannulated to administer antibodies and maintain anesthetic. Following laparotomy, rats were placed in a supine position on an adjustable Plexiglas microscope stage, and a segment of the midjejunum was exteriorized and prepared for intravital microscopy as previously described $(3,4,9)$.

The mesenteric preparation was observed through an intravital microscope (Optiphot-2; Nikon Inc., Mississauga, Ontario, Canada) with a $\times 25$ objective lens (Wetzlar L25/0.35; E. Leitz Inc., Munich, Germany) and a $\times 10$ eyepiece. A video camera (model 5100 HS; Panasonic, Osaka, Japan) mounted on the microscope projected the image onto a color monitor (model PVM 2030; Sony Corp., Tokyo, Japan), and the images were recorded for subsequent playback analysis using a videocassette recorder (model AG-1790; Panasonic). The final magnification of the image on the monitor was $\times 1,800$. Single, unbranched mesenteric venules $(30-50 \mu \mathrm{m}$ in diameter) were selected for study. The same section of venule was observed throughout the experiment to control for variations between different regions. Venular diameter was measured on-line using a video caliper (Microcirculation Research Institute, Texas A\&M University, College Station, Texas, USA). Centerline red blood cell velocity (Vrbc) was also measured on-line using an optical Doppler velocimeter (Microcirculation Research Institute, Texas A\&M University). Mean red blood cell velocity was calculated assuming cylindrical geometry: Vmean $=\mathrm{Vrbc} / 1.6$. Venular wall shear rate $(\gamma)$ was calculated based on the Newtonian definition: $\gamma=$ 8 (Vmean/Dv) (29).

The number of rolling and adherent leukocytes was determined off-line during video playback analysis. Leukocytes were considered adherent to the venular endothelium when they remained stationary for 30 seconds or more. Rolling leukocytes were defined as those white blood cells that moved at a velocity less than the velocity of erythrocytes within the same vessel. The flux of rolling leukocytes was determined as the number of 
a

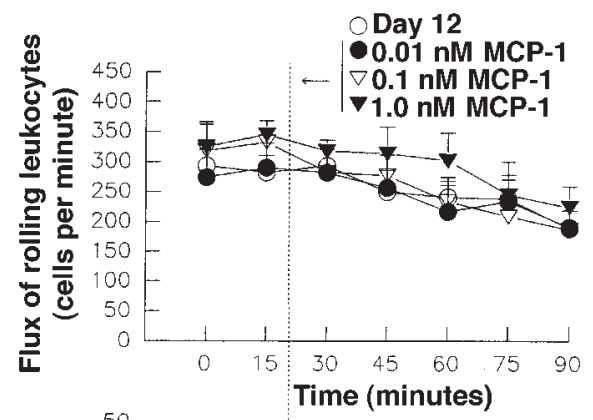

b

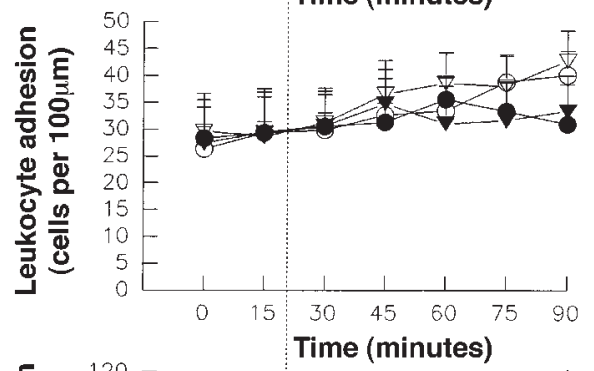

c

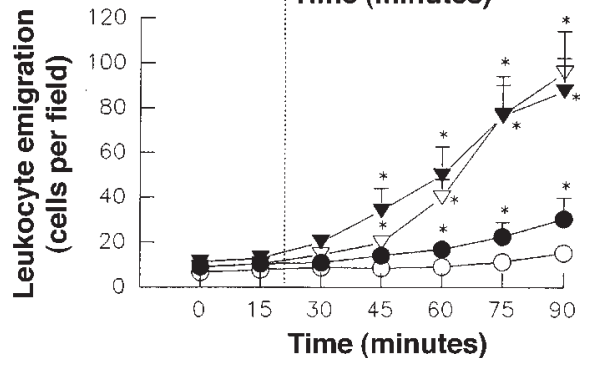

Figure 2

Leukocyte rolling flux (a), adhesion (b), and emigration (c) in mesenteric postcapillary venules of adjuvant-immunized rats superfused with control buffer or the CC chemokine MCP-1. Following an initial 20-minute control period, during which the mesentery was superfused with bicarbonate-buffered saline, the mesentery was superfused for 70 minutes with buffered saline (day 12, $n=6$ ) or buffered saline containing 0.01 ( $n$ $=6), 0.1(n=5)$, or $1.0(n=5) \mathrm{nM} \mathrm{MCP}-1 .{ }^{*} P<0.05$ vs. untreated day 12 adjuvant-immunized group.

white blood cells that rolled past a fixed point in the venule during a 1-minute interval using frame-by-frame video analysis. Leukocyte emigration was measured as the number of leukocytes that could be seen in the field of view $(275 \times 190 \mu \mathrm{m})$ surrounding the venule of interest.

Experimental protocols. We examined leukocyte trafficking through mesenteric postcapillary venules of naive and adjuvantimmunized (12-14 days after immunization) rats. When a mesenteric venule $30-50 \mu \mathrm{m}$ in diameter was located, its image was recorded for 5 minutes. Additional 5-minute recordings were made at 15 -minute intervals over a 90 -minute period and revealed little or no change in hemodynamic parameters or leukocyte kinetics throughout the experiment. Therefore, chemokine and anti-adhesion molecule therapies were administered following 2 baseline recordings (at the 20 -minute time point), so that each animal could serve as its own control for the effects of treatments on leukocyte rolling flux, adhesion, and emigration.

The rat CC chemokine MCP-1 (PeproTech Inc., Rocky Hill, New Jersey, USA) was superfused over the mesentery at concentrations of $0.01,0.1$, or $1.0 \mathrm{nM}$ in buffered saline. The endotoxin content was less than $0.1 \mathrm{ng} / \mu \mathrm{g}$ of rat MCP-1, more than 10,000 fold lower than the dose of endotoxin required to increase leukocyte adhesion in the rat mesentery (30). As $0.1 \mathrm{nM} \mathrm{MCP-1}$ produced optimal emigration in the adjuvant-immunized rats, this concentration was used in further experiments. Immunized animals were treated with antibodies directed against either the $\beta_{2^{-}}$ integrin CD18 (WT-3; $2 \mathrm{mg} / \mathrm{kg}$ ) or the $\alpha_{4}$-integrin CD49d (TA2; $4 \mathrm{mg} / \mathrm{kg}) 15$ minutes prior to superfusion with MCP-1. This concentration of WT-3 has been shown to inhibit leukocyte adhesion induced by the chemotactic peptide $N$-formylmethionyl-leucyl-phenylalanine (fMLP) (31). The TA-2 antibody has been shown to attenuate lymphocyte accumulation during delayed-type hypersensitivity (32) and to block leukocyte rolling and adhesion in adjuvant-immunized rats but not in untreated animals $(3,9)$. To differentiate between mononuclear and polymorphonuclear leukocyte populations, some animals were treated with an anti-neutrophil serum $(0.1 \mathrm{~mL} / \mathrm{rat}$; Accurate Chemical \& Scientific Corp., Westbury, New York, USA). This serum depletes circulating neutrophils without affecting other leukocyte subpopulations (9). Systemic blood samples were obtained at the beginning and end of each experiment for determination of total cell counts and population distributions.

Histology. At the end of some experiments, the mesentery was fixed by superfusion with $5 \%$ glutaraldehyde and then excised from the preparation. Samples were embedded in JB-4 plastic, cut into $2-\mu \mathrm{m}$ sections, and stained with toluidine blue O. Differentiation of recruited cell types was made by a blinded observer based on cellular morphology.

Chemotaxis assays. Leukocytes were isolated from acid-citratedextrose anticoagulated rat blood by a 45 -minute sedimentation over $4 \%$ dextran ( $\mathrm{mol} \mathrm{wt}=250,000$; Spectrum Chemical Manufacturing Corp., Gardena, California, USA) followed by hypotonic lysis of contaminating red blood cells. Leukocytes obtained by this method consisted of more than $85 \%$ neutrophils, with monocytes and lymphocytes making up the remaining populations. Leukocytes were diluted to $6.5 \times$ $10^{6} / \mathrm{mL}$ in HBSS, and $200-\mu \mathrm{l}$ aliquots were added to the top of 6.5 - $\mathrm{mm}$ polyester Transwell inserts $(3-\mu \mathrm{m}$ pore diameter; Corning-Costar Corp., Cambridge, Massachusetts, USA) coated with $1 \%$ gelatin. Inserts were placed in wells containing $600 \mu \mathrm{l}$ of chemotactic stimulus (control buffer or MCP-1). Plates were incubated for 1 hour at $37^{\circ} \mathrm{C}$ to allow transmigration. Transmigrated cells in the lower well were counted using a hemocytometer and differentiated with a Wright-Giemsa staining kit (Star Plex Scientific, Etobicoke, Ontario, Canada). In some experiments, MCP-1 was added to both the well and the insert to disrupt the chemotactic gradient.

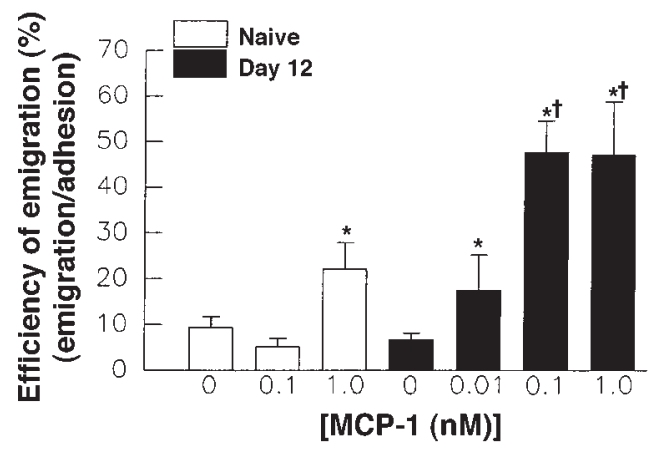

Figure 3

Increased sensitivity of adjuvant-immunized animals to MCP-1 is not due to differences in adhesion. Leukocyte emigration was normalized for differences in adhesion by calculating the percentage of adherent cells that emigrate into the extravascular space ( $n=4-6$ for each experiment). These values represent the average efficiency of emigration for the entire experiment. Although the efficiency of emigration increased over the time course of these experiments, it was higher in adjuvant-immunized rats at all time points (data not shown). ${ }^{*} P<0.05$ vs. no MCP- $1 .+P<0.05$ vs. naive control animals. 
Table 1

Effects of chemokine MCP-1 on hemodynamic parameters in adjuvantimmunized rats

\begin{tabular}{lcccc}
\hline Parameter & No chemokine & $0.01 \mathrm{nM}$ & $0.1 \mathrm{nM}$ & $1.0 \mathrm{nM}$ \\
& & MCP-1 & MCP-1 & MCP-1 \\
Naive: & $n=6$ & ND & $n=6$ & $n=4$ \\
Venular diameter $(\mu \mathrm{m})$ & $39.9 \pm 1.1$ & $\mathrm{ND}$ & $38.3 \pm 1.3$ & $39.5 \pm 1.9$ \\
RBC velocity $(\mathrm{mm} / \mathrm{s})$ & $2.1 \pm 0.4$ & $\mathrm{ND}$ & $2.4 \pm 0.4$ & $2.4 \pm 0.5$ \\
Venular shear rate $\left(\mathrm{s}^{-1}\right)$ & $263 \pm 67$ & $\mathrm{ND}$ & $313 \pm 50$ & $329 \pm 62$ \\
& & & & \\
Day 12 immunized: & $n=6$ & $n=6$ & $n=5$ & $n=5$ \\
Venular diameter $(\mu \mathrm{m})$ & $42.4 \pm 3.7$ & $44.1 \pm 2.0$ & $42.8 \pm 1.7$ & $42.9 \pm 1.4$ \\
RBC velocity $(\mathrm{mm} / \mathrm{s})$ & $2.0 \pm 0.3$ & $2.0 \pm 0.2$ & $1.9 \pm 0.2$ & $2.1 \pm 0.2$ \\
Venular shear rate $\left(\mathrm{s}^{-1}\right)$ & $225 \pm 45$ & $227 \pm 34$ & $222 \pm 34$ & $245 \pm 27$
\end{tabular}

There are no statistically significant differences between groups. Values are the mean of each parameter for a 90-minute experiment. MCP-1 did not cause alterations in hemodynamics over the course of the experiments (data not shown). ND, not done. $\mathrm{RBC}$, red blood cell.

Flow cytometry. Rat leukocytes $\left(1 \times 10^{6}\right)$ were fixed and permeabilized with a cell permeabilization kit (Caltag Laboratories Inc., Burlingame, California, USA). During the permeabilization step, cells were incubated with a control antibody or antibodies against the cytoplasmic tail of rat CCR1 or CCR2 (Santa Cruz Biotechnology Inc., Santa Cruz, California, USA). After 15 minutes at room temperature, cells were washed and stained in the dark for 30 minutes with FITC-labeled mouse anti-goat IgG (Santa Cruz Biotechnology Inc.). Cells were washed and analyzed by FACScan flow cytometry (Becton Dickinson, Mississauga, Ontario). Neutrophil and mononuclear leukocyte populations were gated using forward and side scatter characteristics.

Statistical analysis. All values are reported as means \pm SEM. The data within groups were compared using paired Student's $t$ tests, with Bonferroni corrections for multiple comparisons where appropriate. An unpaired Student's $t$ test was used to compare between groups. Statistical significance was set at $P<0.05$.

\section{Results}

Sensitivity to MCP-1 is increased in a model of adjuvant-induced vasculitis. Figure 1 shows the effects of local MCP- 1 superfusion on leukocyte-endothelium interactions in mesenteric venules of naive rats. Leukocyte rolling flux was not affected by either 0.1 or $1.0 \mathrm{nM}$ MCP-1 (Figure 1a), consistent with previous observations in wild-type and CCR2-deficient mice (16). However, both doses of MCP1 caused increases in the number of firmly adherent leukocytes (Figure 1b). Superfusion with $1.0 \mathrm{nM}$ (but not $0.1 \mathrm{nM}$ ) MCP-1 also caused a small increase in leukocyte migration out of the vasculature (Figure 1c). Histologic examination of the tissue at the end of each experiment revealed few leukocytes, with no discernible differences in cell distribution from control tissues (data not shown).

As MCP-1 function has only been examined previously in naive animals, it is not known how this chemokine will influence leukocyte recruitment in chronic inflammatory disease states. Immunizing rats with $M$. butyricum adjuvant induced a profound vasculitis, with increases in leukocyte rolling flux (250-300 vs. 30-40 cells per minute in control animals) and adhesion (25-35 vs. $2-4$ cells per $100 \mu \mathrm{m}$ venule length in control animals) within mesenteric postcapillary venules (compare Figure 1 with Figure 2 and ref. 3). Despite these large increases in leukocyte trafficking, there was no significant increase in the number of emigrated leukocytes initially present in the extravascular tissue of adjuvantimmunized animals $(8.9 \pm 2.2$ cells per field) compared with naive animals $(5.7 \pm 1.9$ cells per field).

Figure 2 shows the effects of superfusing MCP- 1 onto the mesentery of adjuvant-immunized rats. MCP-1 at concentrations of $0.01,0.1$, and $1.0 \mathrm{nM}$ had no effect on leukocyte rolling flux (Figure 2a) or firm adhesion (Figure $2 \mathrm{~b}$ ). However, superfusion with MCP-1 caused dosedependent increases in the number of emigrated leukocytes (Figure 2c). Maximal emigration was observed with $0.1 \mathrm{nM}$ MCP-1, which induced a net emigration of $85.6 \pm 16.6$ cells per field after 70 minutes of continuous superfusion. This was a significant increase in emigration compared with the naive animals treated with 0.1 or 1.0 nM MCP-1. Superfusion with MCP-1 had no significant effects on hemodynamics, including venular shear rates (Table 1 ).

MCP-1 induced leukocyte emigration in adjuvantimmunized rats at much lower concentrations than in naive animals. To ensure that differences in leukocyte emigration between the naive and adjuvant-immunized rats were not related to differences in the number of firmly adherent cells, leukocyte emigration was normalized for differences in adhesion (Figure 3). Despite higher levels of leukocyte adhesion in the adjuvant-immunized animals, the proportion of adherent neutrophils that emigrated in these animals was increased at all doses of MCP-1 compared with naive rats. Therefore, differences in adhesion cannot account for the increased sensitivity to MCP-1.

Although MCP-1 is a CC chemokine thought to act primarily on monocytes and lymphocytes, histologic assessment of mesenteric tissues revealed that the vast majority of recruited leukocytes were neutrophils (Figure 4 and Table 2). The recruitment of neutrophils was confirmed using an anti-neutrophil serum $(0.1 \mathrm{~mL} / \mathrm{rat})$

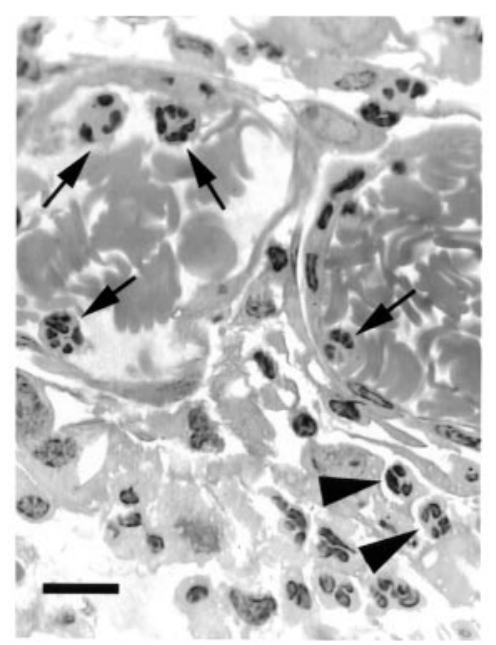

\section{Figure 4}

Neutrophil emigration in the mesentery of an adjuvant-immunized (day 12) rat after 75 minutes of superfusion with $1.0 \mathrm{nM}$ MCP-1. Histologic sections stained with toluidine blue $\mathrm{O}$ show marginated intravascular neutrophils (arrows) and large numbers of extravascular neutrophils (arrowheads). Scale bar: $10 \mu \mathrm{m}$. 
Table 2

Histologic distribution of emigrated leukocytes after MCP-1 superfusion

\begin{tabular}{lccc}
\hline & \multicolumn{3}{c}{$\begin{array}{c}\text { Leukocytes counted in } 5 \\
\text { high-power fields }\left(5 \pm 0.05 \mathrm{~mm}^{2}\right)\end{array}$} \\
$\begin{array}{l}\text { Total } \\
\text { reatment }\end{array}$ & $\begin{array}{c}\text { Neutrophils } \\
\text { teukocytes }\end{array}$ & $\begin{array}{c}\text { Mononuclear } \\
\text { leukocytes }\end{array}$ \\
Buffered saline & 119 & $100(84.0 \%)$ & $19(16.0 \%)$ \\
$.01 \mathrm{nM}$ MCP-1 & 216 & $195(90.3 \%)$ & $21(9.7 \%)$ \\
$.1 \mathrm{nM} \mathrm{MCP-1}$ & 764 & $747(97.8 \%)$ & $17(2.2 \%)$ \\
$.0 \mathrm{nM}$ MCP-1 & 1,112 & $1,095(98.5 \%)$ & $17(1.5 \%)$ \\
\hline
\end{tabular}

that depleted $\sim 90 \%$ of the neutrophils from the circulating blood pool (Table 3) and blocked MCP-1-induced emigration by $\sim 80 \%$ (Table 3 ).

It is possible that MCP-1 may have stimulated extravascular cells, including mast cells and/or macrophages, to release mediators that recruit neutrophils. To determine whether MCP-1 had a direct effect on neutrophils from adjuvant-immunized rats, in vitro chemotaxis assays were performed using isolated rat leukocytes. Figure 5 shows that MCP-1 did not cause chemotaxis of neutrophils from naive rats. However, MCP-1 caused significant chemotaxis of neutrophils from adjuvant-immunized rats at 0.1 and $1.0 \mathrm{nM}$, suggesting a direct chemokine effect on neutrophils. Neutrophils did not migrate in the chemotaxis assay when the chemotactic gradient was disrupted by adding MCP1 to both the upper and lower chambers, suggesting that the response was not due merely to increased chemokinesis. We cannot exclude the possibility that the few contaminating monocytes responded to MCP-1 and then released a factor that recruited neutrophils. However, because there were similar levels of monocyte contamination in leukocyte preparations from control and adjuvant-immunized rats but only neutrophils from adjuvant-immunized rats migrated in response to MCP-1, a role for monocytes is less likely.

Neutrophils in adjuvant-immunized rats express CC chemokine receptors. Flow cytometry was used to characterize the expression of chemokine receptors on leukocytes from naive and adjuvant-immunized rats. Leukocytes were stained with antibodies against the rat MIP- $1 \alpha$ receptor CCR 1 and the MCP-1 receptor CCR2 (Figure 6). Neutrophils from adjuvant-immunized rats, but not naive rats, showed small but consistent increases in log fluorescence intensity when stained with antibodies against CCR1 and CCR2 (Figure 6). The degree of fluorescence seen on neutrophils from adjuvant-immunized animals was of similar magnitude to the fluorescence on positive-staining mononuclear leukocytes from both naive and adjuvant-immunized rats. Although the shifts in fluorescence were small, they are consistent with the low number of MCP-1 binding sites (less than 3,000 per cell) characterized previously on mononuclear leukocytes $(19,33)$.

\section{Table 3} serum
Adhesive mechanisms mediating MCP-1-induced leukocyte emigration. In naive rats, pretreatment with an anti-CD18 mAb (WT-3; $2 \mathrm{mg} / \mathrm{kg}$ intravenously) completely blocked MCP1 -induced increases in leukocyte adhesion ( $2.3 \pm 1.0$ vs. $11.5 \pm 1.9$ cells per $100 \mu \mathrm{m}$ venule length; $P<0.05)$ and net emigration ( $1.6 \pm 1.2$ vs. $12.2 \pm 2.7$ cells per field; $P<0.05)$. However, in adjuvant-immunized rats, a different adhesive profile was observed. We have previously demonstrated that the $\alpha_{4}$-integrin, rather than CD18, mediates the majority of increased adhesion in adjuvant-immunized rats $(3,9)$. In the presence of $0.1 \mathrm{nM} \mathrm{MCP}-1$, the anti- $\alpha_{4-}$ integrin $\mathrm{mAb}$ (TA-2; $4 \mathrm{mg} / \mathrm{kg}$ intravenously) significantly reduced leukocyte firm adhesion, whereas treatment with anti-CD18 mAb (WT-3; $2 \mathrm{mg} / \mathrm{kg}$ intravenously) had no significant effect on leukocyte adhesion (Figure 7a). However, treatment with either antibody reduced MCP1 -induced leukocyte emigration back to baseline levels (Figure 7b). The CD18 antibody appeared to block emigration specifically, as there was no reduction in leukocyte adhesion with this treatment. However, treatment with antibody against the $\alpha_{4}$-integrin reduced both adhesion and emigration. When emigration was normalized for adhesion (Figure 7c), it was found that the few leukocytes that do adhere in the presence of $\alpha_{4}$-integrin antibody emigrate from the vasculature as efficiently as in preparations exposed to MCP-1 in the absence of antibody. This suggests that the $\alpha_{4}$-integrin blocks leukocyte emigration indirectly by reducing leukocyte adhesion. In contrast, the proportion of adherent cells that emigrated in the presence of CD18 antibody was significantly attenuated, suggesting that CD18 is directly involved in the process of transendothelial migration in response to MCP-1.

\section{Discussion}

Using in vitro chemotaxis assays, it has been shown that the CC chemokine MCP-1 will cause chemotaxis of monocytes and lymphocytes but not neutrophils (17-19). Similarly, when injected into the skin or peritoneum of naive rats or mice, MCP-1 elicited primarily monocyte, and some lymphocyte, recruitment (20-22). To our knowledge, this study is the first to demonstrate chemokine responsiveness in a model with a preexisting inflammatory condition. Rats with adjuvant-induced vasculitis had increased sensitivity to MCP-1; leukocyte emigration was induced with a dose of MCP-1 that was 100 -fold lower than the dose required to elicit emigration in naive rats. In addition, the selectivity of MCP-1

Cell counts and net emigration in adjuvant-immunized animals treated with anti-neutrophil

\begin{tabular}{lcccc}
\hline & $\begin{array}{c}\text { Total count } \\
\left(\times 10^{5} / \mathrm{m}\right)\end{array}$ & $\begin{array}{c}\text { PMN count } \\
\left(\times 10^{5} / \mathrm{m}\right)\end{array}$ & $\begin{array}{c}\text { PBMC count } \\
\left(\times 10^{5} / \mathrm{m}\right)\end{array}$ & $\begin{array}{c}\text { Net emigration } \\
\text { (cells per field) }\end{array}$ \\
$\begin{array}{l}\text { Day 12 + 0.1 nM MCP-1 } \\
135.6 \pm 12.6\end{array}$ & $98.8 \pm 9.9$ & $38.3 \pm 3.8$ & $85.6 \pm 16.6$ \\
$\begin{array}{l}+ \text { anti-neutrophil serum } \\
(0.1 \mathrm{~mL} / \mathrm{rat})\end{array}$ & $38.0 \pm 4.5^{\mathrm{A}}$ & $7.8 \pm 1.4^{\mathrm{A}}$ & $30.2 \pm 5.1$ & $17.5 \pm 4.6^{\mathrm{A}}$ \\
& & & &
\end{tabular}

Blood counts and differentials were determined during a 20-minute baseline period and 70 minutes after superfusion with MCP-1. Anti-neutrophil serum was administered in some animals immediately following the baseline period. Net emigration into the extravascular space was determined after 70 minutes of MCP-1 superfusion. ${ }^{A} P<0.05$ vs. chemokine treatment alone. 


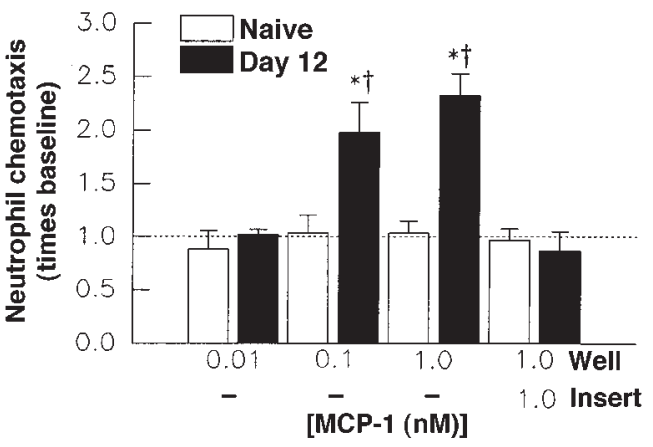

Figure 5

MCP-1-induced neutrophil chemotaxis in vitro. Neutrophils isolated from control or adjuvant-immunized rats were placed in filter inserts $(3-\mu \mathrm{m}$ pores) over wells containing $0.01,0.1$, or $1.0 \mathrm{nM} \mathrm{MCP-1}$. In one group, $1.0 \mathrm{nM} \mathrm{MCP}-1$ was added to both the well and the insert in order to disrupt the chemotactic gradient. After a 60 -minute incubation, the proportion of neutrophils that migrated into the lower chamber was calculated. All values were normalized compared with basal migration in the absence of chemotactic stimulus ( $n=4-6$ separate experiments for each data group). ${ }^{*} P<0.05$ vs. basal migration. ${ }^{\dagger} P<0.05$ vs. naive control rats.

for mononuclear leukocyte subsets was altered; MCP-1 was able to recruit neutrophils from adjuvant-immunized rats, but not from naive animals, in vivo and in vitro. This phenotype was associated with an upregulation of the chemokine receptors CCR1 and CCR2 in neutrophils from adjuvant-immunized rats. These results suggest that chemokine responsiveness can be altered in chronic inflammatory disease states.

Other investigators have suggested previously that MCP-1 may activate a secondary cascade of inflammatory mediators, which leads to neutrophil recruitment in vivo. This argument was used to explain the observation that anti-MCP-1/JE mAb's could reduce neutrophil recruitment to sites of Cryptococcus neoformans, thioglycollate, and TNF- $\alpha$-induced inflammation in the mouse $(21,22,34)$. However, treatment with MCP-1 itself did not induce neutrophil recruitment in the hands of these investigators $(21,22)$, suggesting that MCP-1-associated recruitment of neutrophils may involve complex interactions in vivo. It is possible that MCP-1 could interact with other inflammatory mediators to activate mast cells or tissue resident macrophages, causing them to release mediators that induce neutrophil recruitment. Although we cannot rule out indirect recruitment of neutrophils by MCP-1 in adjuvant-immunized rats in vivo, the increased responsiveness of these neutrophils to MCP- 1 in vitro and the induction of CCR 2 strongly suggest a direct effect of MCP-1 on neutrophils from adjuvant-immunized rats.

MCP-1 has been shown to bind to 3 different chemokine receptors: CCR1, CCR2, and CCR4 (26, 27, $35,36)$. While CCR2 and CCR 4 can mediate leukocyte responsiveness and chemotaxis to MCP-1, CCR1 does not transduce strong secondary signals in response to MCP-1 binding in vitro (36). Although CCR2 expression has not been detected in human neutrophils (37), and the receptor was not expressed on normal rat neutrophils, flow cytometry data demonstrated that CCR2 was upregulated in circulating neutrophils from adju- vant-immunized rats. This provides a direct mechanism to explain the increased neutrophil responsiveness to MCP-1 in vitro and in vivo. It is also possible that neutrophils may upregulate signaling through CCR1 or increase expression of other MCP-1 receptors such as CCR4 during adjuvant-induced vasculitis. Recent data have highlighted the fact that the inflammatory microenvironment may cause neutrophils to upregulate CC chemokine receptors. For example, IFN- $\gamma$ has been shown to upregulate functional expression of CCR1 and CCR3 on human neutrophils in vitro (37). It is conceivable that endogenous cytokines or other factors may upregulate neutrophil responsiveness to MCP-1 in adjuvant-immunized rats.

\section{Naive control rats}

\section{Neutrophils}
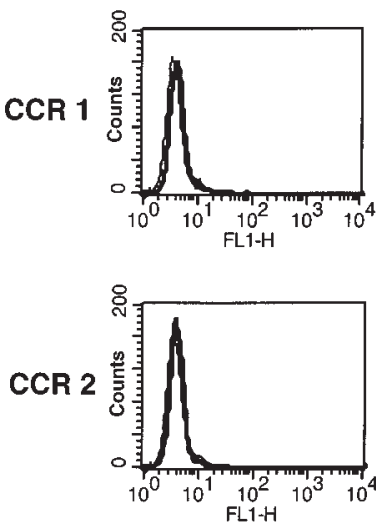

Adjuvant-immunized rats Neutrophils
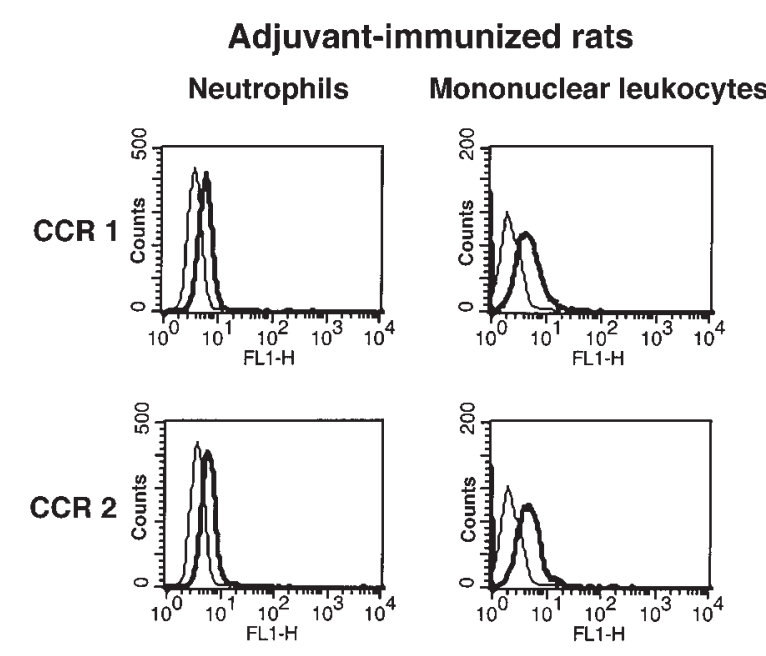

\section{Mononuclear leukocytes}
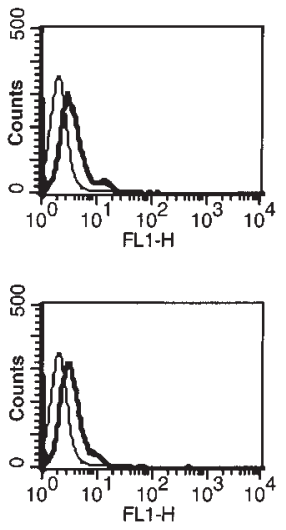

Figure 6

Expression of chemokine receptors on rat leukocytes. The flow cytometry histograms show log fluorescence intensity of CCR 1 and CCR2 on rat neutrophils and mononuclear leukocytes from naive control and adjuvant-immunized rats. Gating on mononuclear leukocytes stained with CCR1 and CCR2 antibodies shifted the log fluorescence intensity to the right compared with a control antibody in both groups. Gating on neutrophils stained with CCR1 and CCR2 antibodies produced a shift to the right only in neutrophils from adjuvant-immunized rats. Histograms are representative of 5 animals in each group. 
a

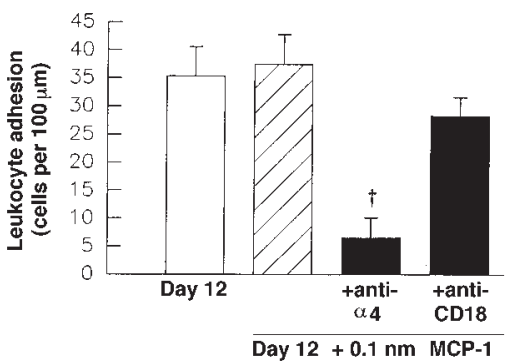

b

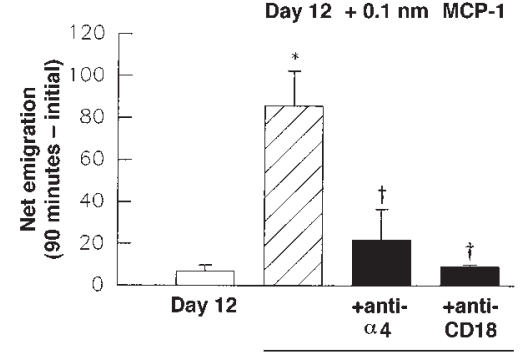

C

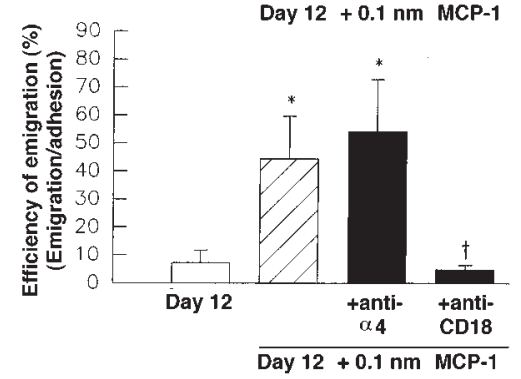

Figure 7

Role of CD18 and the $\alpha_{4}$-integrin in MCP-1-induced leukocyte adhesion and transmigration in adjuvant-immunized rats. Rats were treated with an anti- $\alpha_{4}$-integrin mAb (TA-2; $\left.4 \mathrm{mg} / \mathrm{kg}, n=5\right)$ or an anti-CD18 mAb (WT-3; $2 \mathrm{mg} / \mathrm{kg}$ intravenously, $n=4) 15$ minutes prior to superfusion with $0.1 \mathrm{nM}$ MCP-1. Values represent (a) adhesion in postcapillary venules and (b) emigration into the extravascular space after 70 minutes of MCP-1 superfusion. (c) Leukocyte emigration was normalized for differences in adhesion by calculating the percentage of adherent cells that emigrate into the extravascular space. ${ }^{*} P<0.05$ vs. untreated day 12 adjuvant-immunized group. ${ }^{\dagger} P<0.05$ vs. $0.1 \mathrm{nM} \mathrm{MCP-1}$ treatment.

Additional changes in neutrophil responsiveness are evident in the observation that an $\alpha_{4}$-integrin antibody could block a large proportion of the chemokine-induced neutrophil emigration in the adjuvant-induced vasculitis model. This suggests that neutrophils in this model primarily used $\alpha_{4}$-integrin-dependent mechanisms to adhere to the endothelium. Even though the $\alpha_{4}$-integrin can be detected at low levels on rat neutrophils (38), there has been no role found for the $\alpha_{4}$-integrin in mediating neutrophil-endothelial cell interactions under baseline conditions or in acute models of inflammation (leukotriene $\mathrm{C}_{4}$, LPS less than 90 minutes) $(3,9)$. However, the $\alpha_{4}$-integrin has been shown to mediate neutrophil accumulation in the joints and at other inflammatory sites in adjuvant-immunized rats $(3,9,38)$, presumably because of the upregulation of an endothelial ligand for the $\alpha_{4}$-integrin. A recent study by Chen et al. (39) suggests that the $\alpha_{4}$-integrin is expressed on leukocytes in various activation states and that a certain high-affinity subset of the $\alpha_{4}$-integrin can mediate spontaneous adhesion to $\alpha_{4^{-}}$ integrin ligands in the absence of an external stimulus. This may explain the spontaneous leukocyte adhesion observed in adjuvant-immunized rats; yet, it is unclear why MCP-1 did not increase adhesion in these animals. Perhaps these cells are already partly activated and the MCP-1 signal was redundant for adhesion. However, the chemotactic gradient was required to direct the migration of these cells in our assay.

To our knowledge, our data provide the first direct functional evidence that CD18 is important in emigration in vivo. Previous studies have demonstrated that platelet-endothelial cell adhesion molecule-1 (PECAM1), a molecule found on both leukocytes and endothelium, is important in mediating some leukocyte transendothelial migration in vitro and in vivo $(40,41)$. However, it has been very difficult to determine whether the integrins play important roles in transendothelial migration, as they are critical in mediating firm adhesion to the endothelium, an upstream adhesive interaction. This study was able to dissociate these two functions in the adjuvant-induced vasculitis model. In adjuvantimmunized rats, the $\alpha_{4}$-integrin mediated the majority of firm adhesion in the presence of MCP-1. In contrast, CD18 did not contribute to the adhesion observed in this model, but CD18 blockade significantly reduced emigration in response to MCP-1. These data show that CD18 is important in mediating leukocyte transendothelial migration in vivo.

In other studies, integrin cross-talk has been shown to regulate adhesive interactions. For example, $\beta_{2}$-integrin binding can downregulate $\alpha_{4} \beta_{1}$-integrin-dependent adhesion (42). This may be important in allowing cells adhering by the $\alpha_{4}$-integrin to migrate through the vascular endothelium via a CD18-dependent mechanism. Similarly, the $\alpha_{v} \beta_{3}$-integrin, which binds PECAM- 1 and vitronectin, has been shown to enhance the rate of migration through monolayers expressing intercellular adhesion molecule-1 (ICAM-1) and lymphocyte migration on vascular cell adhesion molecule-1 (VCAM-1) (43, 44). Whether additional adhesion molecules, such as the $\alpha_{v} \beta_{3}$-integrin, are involved in regulating the migration process in adjuvant-immunized rats remains to be determined. However, it is likely that additional factors, other than chemokine responsiveness of leukocytes, influence emigration in adjuvant-immunized rats. For example, the CXC chemokine CINC/gro also induced enhanced leukocyte emigration in adjuvant-immunized rats but did not exhibit increased neutrophil chemotaxis in vitro (Johnston, B., et al., unpublished observations). This suggests that the rate of migration may also be influenced by the endothelium and the adhesion molecules that are expressed during adjuvant-induced vasculitis.

In conclusion, this study found that MCP-1 was more effective at recruiting leukocytes in a model of chronic inflammation than in healthy tissue, suggesting that the relevant effective concentration of this inflammatory mediator may be lower during disease states than anticipated from previous studies. Additionally, the specificity of chemokines for certain leukocyte subsets may change during chronic inflammation, as neutrophils were able to upregulate receptors for the CC chemokines. These studies highlight the importance of understanding the mechanisms that operate under chronic inflammatory conditions, because responses 
elicited by chemokines and other inflammatory mediators may be altered in disease states compared with the responses elicited in healthy individuals.

\section{Acknowledgments}

The authors thank Mirjana Maric for her valuable assistance with the flow cytometry assays. This work was supported by a grant from the Medical Research Council of Canada (MRC) (to P. Kubes) and by grants from the Methodist Hospital Foundation and the National Institutes of Health (HL-42550) (to A.R. Burns). P. Kubes is an MRC Scientist and a Scientist of the Alberta Heritage Foundation for Medical Research (AHFMR). A.R. Burns is the recipient of a Chao fellowship. B. Johnston is the recipient of an AHFMR studentship.

1. Springer, T.A. 1994. Traffic signals of lymphocyte recirculation and leukocyte emigration: the multistep paradigm. Cell. 76:301-314.

2. Von Andrian, U.H., et al. 1991. Two-step model of leukocyte-endothelial cell interaction in inflammation: distinct roles for LECAM-1 and the leukocyte $\beta 2$ integrins in vivo. Proc. Natl. Acad. Sci. USA. 88:7538-7542.

3. Johnston, B., et al. 1997. Differential roles of the selectins and the a4integrin in acute, subacute, and chronic leukocyte recruitment in vivo. J. Immunol. 159:4514-4523.

4. Kubes, P., and Kanwar, S. 1994. Histamine induces leukocyte rolling in postcapillary venules: a P-selectin-mediated event. J. Immunol. 152:3570-3577.

5. Kanwar, S., et al. 1997. The association between $\alpha 4$-integrin, P-selectin, and E-selectin in an allergic model of inflammation. J. Exp. Med. 185:1077-1087.

6. Sriramarao, P., Von Andrian, U.H., Butcher, E.C., Bourdon, M.A., and Broide, D.H. 1994. L-selectin and very late antigen-4 integrin promote eosinophil rolling at physiological shear rates in vivo. J. Immunol. 153:4238-4246.

7. Luscinskas, F.W., et al. 1994. Monocyte rolling, arrest and spreading on IL-4-activated vascular endothelium under flow is mediated via sequential action of L-selectin, $\beta 1$-integrins, and $\beta 2$-integrins. J. Cell Biol. 125:1417-1427.

8. Alon, R., et al. 1995. The integrin VLA-4 supports tethering and rolling in flow on VCAM-1. J. Cell Biol. 128:1243-1253.

9. Johnston, B., Issekutz, T.B., and Kubes, P. 1996. The $\alpha 4$-integrin supports leukocyte rolling and adhesion in chronically inflamed postcapillary venules in vivo. J. Exp. Med. 183:1995-2006.

10. Suzuki, H., et al. 1994. Rat CINC/gro: a novel mediator for locomotive and secretagogue activation of neutrophils in vivo. J. Leukoc. Biol. 55:652-657.

11. Hakansson, L., and Venge, P. 1990. Inhibition of neutrophil and eosinophil chemotactic responses to PAF by the PAF-antagonists WEB2086, L652,731, and SRI-63441. J. Lenkoc. Biol. 47:449-456.

12. Palmbald, J., et al. 1981. Leukotriene B4 is a potent and stereospecific stimulator of neutrophil chemotaxis and adherence. Blood. 58:658-661.

13. Gao, J.-X., and Issekutz, A.C. 1996. Mac-1 (CD11b/CD18) is the predominant $\beta 2$ (CD18) integrin mediating human neutrophil migration through synovial and dermal fibroblast barriers. Immunology. 88:463-470.

14. Ohashi, K.L., Tung, D.K.-L., Zweifach, B.W., and Schmid-Schönbein, G.W. 1996. Transvascular and interstitial migration of neutrophils in rat mesentery. Microcirculation. 3:199-210.

15. Rollins, B.J. 1997. Chemokines. Blood. 90:909-928.

16. Kuziel, W.A., et al. 1997. Severe reduction in leukocyte adhesion and monocyte extravasation in mice deficient in CC chemokine receptor 2. Proc. Natl. Acad. Sci. USA. 94:12053-12058.

17. Valente, A.J., Graves, D.T., Vialle-Valentin, C.E., Delgado, R., and Schwartz, C.J. 1988. Purification of a monocyte chemotactic factor secreted by nonhuman primate vascular cells in culture. Biochemistry. 27:4162-4168.

18. Loetscher, P., Seitz, M., Clark-Lewis, I., Baggiolini, M., and Moser, B. 1994. Monocyte chemotactic proteins MCP-1, MCP-2, and MCP-3 are major attractants for human CD4+ and CD8+ T lymphocytes. FASEB J. 8:1055-1060

19. Ernst, C.A., et al. 1994. Biochemical and biologic characterization of murine monocyte chemoattractant protein-1: identification of two functional domains. J. Immunol. 152:3541-3549.
20. Zachariae, C.O.C., et al. 1990. Properties of monocyte chemotactic and activating factor (MCAF) purified from a human fibrosarcoma cell line. J. Exp. Med. 171:2177-2182.

21. Tessier, P.A., et al. 1997. Chemokine networks in vivo: involvement of C$\mathrm{X}-\mathrm{C}$ and $\mathrm{C}-\mathrm{C}$ chemokines in neutrophil extravasation in vivo in response to TNF- $\alpha$. J. Immunol. 159:3595-3602.

22. Ajuebor, M.N., et al. 1998. Endogenous monocyte chemoattractant protein-1 recruits monocytes in the zymosan peritonitis model. J. Leukoc. Biol. 63:108-116.

23. Gunn, M.D., Nelken, N.A., Liao, X., and Williams, L.T. 1997. Monocyte chemoattractant protein- 1 is sufficient for the chemotaxis of monocytes and lymphocytes in transgenic mice but requires an additional stimulus for inflammatory activation. J. Immunol. 158:376-383.

24. Fuentes, M.E., et al. 1995. Controlled recruitment of monocytes and macrophages to specific organs through transgenic expression of monocyte chemoattractant protein-1. J. Immunol. 155:5769-5776.

25. Frade, J.M.R., et al. 1997. Characterization of the CCR2 chemokine receptor: functional characterization of CCR2 receptor expression on $\mathrm{B}$ cells. J. Immunol. 159:5576-5584.

26. Kurihara, T., and Bravo, R. 1996. Cloning and functional expression of mCCR2, a murine receptor for the C-C chemokines JE and FIC. J. Biol. Chem. 271:11603-11606.

27. Charo, I.F., et al. 1994. Molecular cloning and functional expression of two monocyte chemoattractant protein 1 receptors reveals alternative splicing of the carboxyl-terminal tails. Proc. Natl. Acad. Sci. USA. 91:2752-2756.

28. Boring, L., et al. 1997. Impaired monocyte migration and reduced type 1 (Th1) cytokine responses in C-C chemokine receptor 2 knockout mice. J. Clin. Invest. 100:2552-2561.

29. House, S.D., and Lipowsky, H.H. 1987. Leukocyte-endothelium adhesion: microhemodynamics in mesentery of the cat. Microvasc. Res. 34:363-379.

30. Davenpeck, K.L., Steeber, D.A., Tedder, T.F., and Bochner, B.S. 1997. Pand $\mathrm{L}$-selectin mediate distinct but overlapping functions in endotoxininduced leukocyte-endothelial interactions in the rat mesenteric microcirculation. J. Immunol. 159:1977-1986.

31. Suematsu, M., et al. 1994. Microvascular oxidative stress preceding leukocyte activation elicited by in vivo nitric oxide suppression. Am.J. Physiol. 266:H2410-H2415.

32. Issekutz, T.B. 1991. Inhibition of in vivo lymphocyte migration to inflammation and homing to lymphoid tissues by the TA- 2 monoclonal antibody: a likely role for VLA-4 in vivo. J. Immunol. 147:4178-4184.

33. Yoshimura, T., and Leonard, E.J. 1990. Identification of high affinity receptors for human monocyte chemoattractant protein-1 on human monocytes. J. Immunol. 145:292-297.

34. Huffnagle, G.B., et al. 1995. The role of monocyte chemotactic protein-1 (MCP-1) in the recruitment of monocytes and $\mathrm{CD}^{+} \mathrm{T}$ cells during a pulmonary Cryptococcus neoformans infection. J. Immunol. 155:4790-4797.

35. Power, C.A., et al. 1995. Molecular cloning and functional expression of a novel CC chemokine receptor cDNA from a human basophilic cell line. J. Biol. Chem. 270:19495-19500.

36. Neote, K., DiGregorio, D., Mak, J.Y., Horuk, R., and Schall, T.J. 1993. Molecular cloning, functional expression, and signalling characteristics of a C-C chemokine receptor. Cell. 82:415-425.

37. Bonecchi, R., et al. 1999. Up-regulation of CCR1 and CCR3 and induction of chemotaxis to CC chemokines by IFN-gamma in human neutrophils. J. Immunol. 162:474-479.

38. Issekutz, T.B., Miyasaka, M., and Issekutz, A.C. 1996. Rat blood neutrophils express very late antigen 4 and it mediates migration to arthritic joint and dermal inflammation. J. Exp. Med. 183:2175-2184.

39. Chen, C., et al. 1999. High affinity very late antigen-4 subsets expressed on $\mathrm{T}$ cells are mandatory for spontaneous adhesion strengthening but not for rolling on VCAM-1 in shear flow. J. Immunol. 162:1084-1095.

40. Muller, W.A., Weigl, S.A., Deng, X., and Phillips, D.M. 1993. PECAM-1 is required for transendothelial migration of leukocytes. J. Exp. Med. 178:449-460.

41. Bogen, S., Pak, J., Garifallou, M., Deng, X., and Muller, W.A. 1994. Monoclonal antibody to murine PECAM-1 (CD31) blocks acute inflammation in vivo. J. Exp. Med. 179:1059-1064.

42. Porter, J.C., and Hogg, N. 1997. Integrin cross talk: activation of lymphocyte function-associated antigen-1 on human T cells alters $\alpha 4 \beta 1$ and $\alpha 5 \beta 1$-mediated function. J. Cell Biol. 138:1437-1447.

43. Imhof, B.A., et al. 1997. Cross talk between $\alpha v \beta 3$ and $\alpha 4 \beta 1$ integrins regulates lymphocyte migration on vascular cell adhesion molecule 1. Eur. J. Immunol. 27:3242-3252.

44. Weerasinghe, D., et al. 1998. A role for the $\alpha v \beta 3$ integrin in the transmigration of monocytes. J. Cell Biol. 142:595-607. 